\title{
Extension to individual TCP transmission of instantaneous throughput based on occupied duration
}

\author{
Kenji Kita $^{1, *, a)}$, Masato Uchida ${ }^{2}$, Akiharu Tokutake ${ }^{2}$, \\ Hiroyasu Ishikawa ${ }^{3}$, and Hideyuki Shinonaga ${ }^{1,2}$ \\ ${ }^{1}$ Faculty of Science and Engineering, Toyo University \\ 2100 Kujirai, Kawagoe, Saitama 350-8585, Japan \\ ${ }^{2}$ Graduate School of Science and Engineering, Toyo University \\ 2100 Kujirai, Kawagoe, Saitama 350-8585, Japan \\ ${ }^{3}$ College of Engineering, Nihon University \\ 1 Nakagawara, Tokusada, Tamuramachi, Koriyama, Fukushima 963-8642, Japan \\ * Present affiliation: School of Informatics, Daido University \\ a) k-kita@daido-it.ac.jp
}

\begin{abstract}
The instantaneous throughput is evaluated based on the communication traffic volume per unit time. Although the method can roughly evaluate that throughput within a unit time, it is difficult to evaluate the communication situation of each packet in detail. Therefore, the authors have proposed the method of deriving instantaneous throughput based on the occupied duration in individual UDP transmission. In the method, not only instantaneous throughput per packet but also that of other signals such as beacon signals can be evaluated. In this paper, we define and extend the occupied duration in individual TCP transmission, and show an example of analysis compared with the conventional methods.
\end{abstract}

Keywords: radio LAN, occupied duration, instantaneous throughput, TCP, packet capture analysis

Classification: Network

\section{References}

[1] K. Kita, M. Uchida, H. Ishikawa, and H. Shinonaga, "Novel throughput evaluation method of instantaneous throughput of radio LAN systems based on occupied duration for individual UDP transmission," IEICE Commun. Express, vol. 8, no. 1, pp. 7-12, Jan. 2019. DOI: 10.1587/comex.2018xb10118

[2] M. Morikura and S. Kubota, 802.11 High-speed Wireless LAN Textbook, Impress Groupe Company, Tokyo, 2010.

[3] A. Vasan and U. Shankar, "An empirical characterization of instantaneous throughput in 802.11b WLANs," Tech. Rep. CS-TR-4389, UMIACS-TR-200269, Department of Computer Science and UMIACS, University of Maryland College Park, 2002.

[4] SOURCEFORGE, "iperf2," https://sourceforge.net/projects/iperf2/, accessed Aug. 24, 2020. 
[5] riverbed, "riverbed," https://www.riverbed.com/jp/, accessed Aug. 24, 2020.

[6] The Wireshark team, "Wireshark," https://www.wireshark.org/, accessed Aug. 24,2020

\section{Introduction}

The instantaneous throughput based on the communication traffic volume per unit time is evaluated frequently as a communication quality evaluation method. However, it is difficult to evaluate accurately the communication situation. Therefore, we have proposed the throughput evaluation method based on the occupied duration of one packet during the individual UDP transmission [1]. The study has shown that more detailed throughput evaluation with focusing on each packet is possible compared with the conventional method. In this paper, as the simplest case of $802.11 \mathrm{~g}$, we extend the case of TCP transmission by newly defining the case where TCP acknowledgement interrupts at retransmission, and an example applied to actual captured data are indicated.

\section{Definition of throughput based on occupied duration in individual TCP transmission}

An occupied duration in the individual TCP transmission is defined in detail by extending from that of the individual UDP transmission.

\subsection{Conventional instantaneous throughput}

Fig. 1 (a) shows the definition of the instantaneous throughput evaluated by the amount of data received per unit time $t$ seconds. Since this throughput is based on a fixed duration, we call it Fixed Duration $(F D)$ throughput in this study. For example, if 1500 bytes TCP Data exist $N$ packets in $\tau$ seconds, FD throughput is obtained by the following [2]:

$$
\text { FD Throughput (Mbit/s) }=\frac{1500 \times 8 \times N \times 10^{-6}}{\tau} \text {. }
$$

As another method in Fig. 1 (b), a previous study [3] has proposed a method of calculating the throughput based on the duration transmitting a fixed number of $K$ packets, we call it Fixed Number $(F N)$ throughput. Let the start time of the first packet be $t$ and the end time of the K-th packet be $s$, then $\mathrm{K}$ packet transmitting duration is $s-t$. As above, FN throughput is as follows:

$$
\text { FN Throughput (Mbit/s) }=\frac{1500 \times 8 \times K \times 10^{-6}}{s-t} \text {. }
$$

When $K=1$, FN throughput is per individual packet. TCP Acknowledgement (ACK) is also calculated with the same equations using its payload size, generally 52 bytes [2]. 


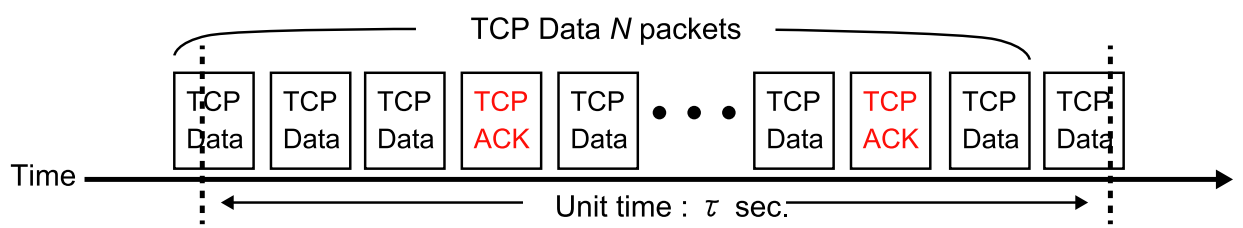

(a) Definition of conventional throughput

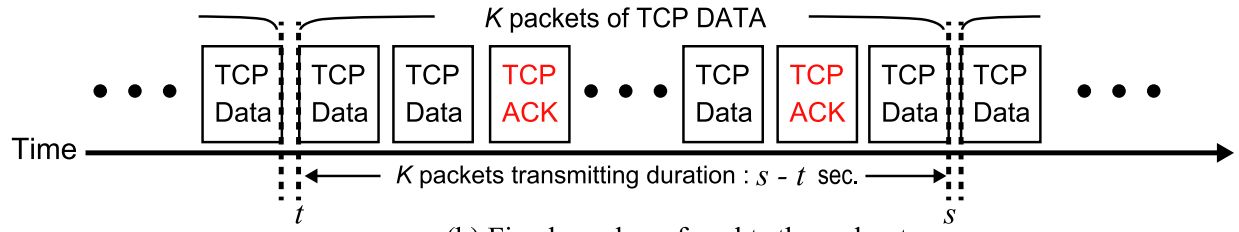

(b) Fixed number of packts throughput

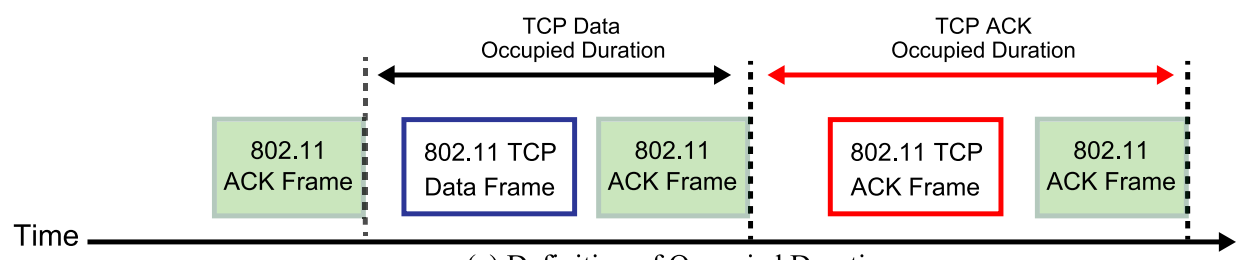

(c) Definition of Occupied Duration

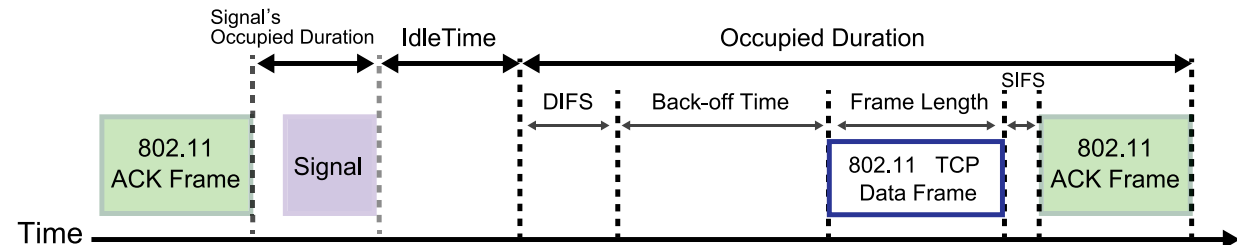

(d) Definition of Occupied Duration when other signal exists

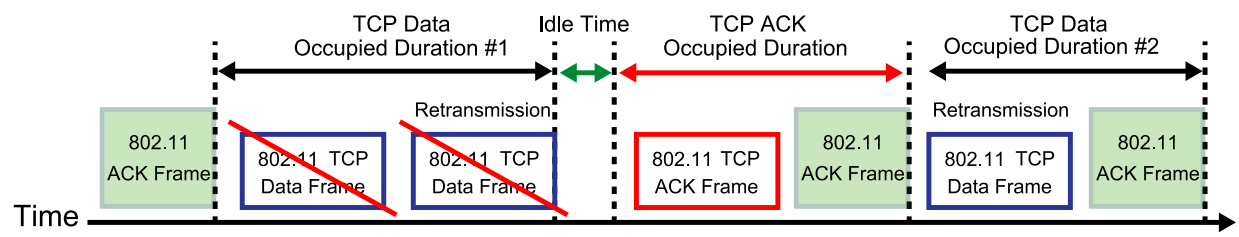

(e) Example of divided Occupied Duration

Fig. 1. Definition of occupied duration.

\subsection{Definition of occupied duration for individual TCP transmission}

The authors have proposed Occupied Duration when one packet occupies a communication path, and Occupied Duration (OD) throughput based on it have been shown in individual UDP transmission [1]. The definition of Occupied Duration is extended so that OD throughput can be derived in the individual TCP transmission as shown Fig. 1 (c). Therefore, Occupied Duration is defined not only for 802.11 TCP Data Frame (TCP Data) but for 802.11 TCP ACK Frame (TCP ACK), and OD throughput for each TCP Data or TCP ACK should be derived. Occupied Duration is defined as from the capture completion time of the preceding 802.11 ACK Frame (ACK Frame) to that of ACK Frame of the target packet. Basically, it is the same as in the individual UDP transmission [1], OD throughput is obtained by the following:

$$
\text { OD Throughput }(\mathrm{Mbit} / \mathrm{s})=\frac{\text { Payload }}{\text { Occupied Duration }},
$$

where Payload is a data size excluding the two physical layer headers of 802.11 and the logical link control header [2]. 


\subsection{Definition of occupied duration when other signals exist}

OD throughput of TCP Data and TCP ACK are derived more accurately than the conventional method by considering Occupied Duration of other signal (Signal) such as beacon signals transmitted by other access points. Fig. 1 (d) shows the definition of Occupied Duration when other signals exist. Its definition is the same as in the UDP individual transmission [1]. Briefly, since Signal does not have ACK Frame, Occupied Duration of TCP Data or TCP ACK transmitted immediately after that is expressed using the average value of Back-off Time. This is because Back-off Time by CSMA/CA differs each packet and it is difficult to measure its value. In this case, there may be Idle Time which is no signal duration between Signal and TCP Data or TCP ACK. Furthermore, the start time of Occupied Duration may be before the capture completion time of Signal. In this case, that of Occupied Duration is set as the capture completion time of Signal. In addition, the Signal's Occupied Duration is also defined, and its OD throughput can be derived.

\subsection{Definition of divided occupied duration}

In rare cases in TCP transmissions, interruptions may occur while other devices are sending and retransmitting packets. An example is shown in Fig 1 (e). TCP Data is retransmitted once and interrupted by TCP ACK. After that, retransmitted the same TCP Data, its ACK Frame is captured normally. In this case, the duration from the capture completion time of the preceding ACK Frame to that of the first retransmission packet is set as TCP Data Occupied Duration \#1. Next, the duration from the ACK Frame capture completion time of the TCP ACK to that of the TCP Data retransmitted again is set as TCP Data Occupied Duration \#2. In this way, Occupied Duration of the same packet is divided, and TCP Data Occupied Duration \#1 and \#2 are summed up to be its Occupied Duration, and OD throughput is calculated using it. Since OD throughput is calculated based on the duration occupying the communication path, when shown it in a figure, it is divided in Occupied Durations. As a result, the usage status of the communication path is visualized. General formula of OD throughput is obtained by the following:

$$
\text { OD Throughput }(\mathrm{Mbit} / \mathrm{s})=\frac{\text { Payload }}{\sum_{k=1}^{N} \text { Occupied Duration } \# k} \text {. }
$$

In addition, considering the preceding packet without ACK Frame as TCP ACK in Fig. 1(e), its Occupied Duration applies the definition of that when other signals exist in Sect. 2.3. In this method, by precisely determining Occupied Duration of one packet, it is possible to accurately analyze the communication situation at the time of packet transmission from OD throughput.

\section{Application example in individual TCP transmission}

\subsection{Experiment system}

Fig. 2 shows an experiment system. TCP Data packets are transmitted from Tx Personal Computer (PC) installed iperf version 2.0.5 [4], which is packet generation software, to Rx PC. TCP ACK packets are transmitted from Rx PC to Tx PC. A capture PC with AirPcap Nx [5] attached was used to capture packets in the radio 


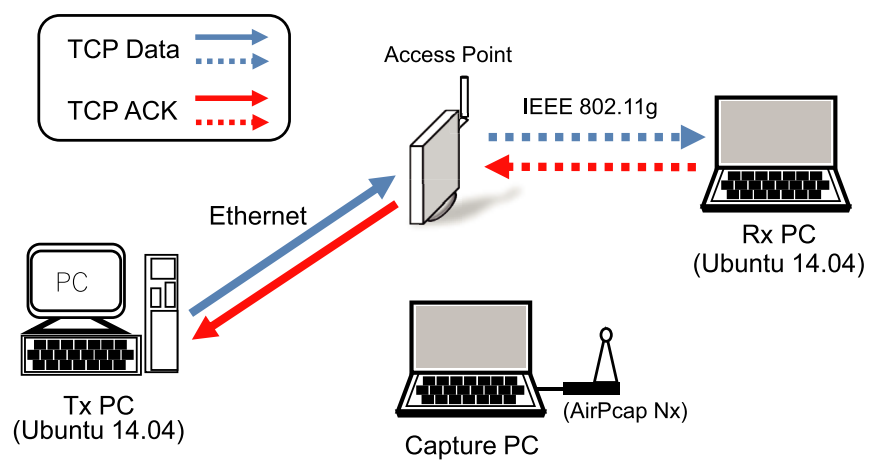

Fig. 2. Experiment system.

section. Wireshark [6] is used for packet analysis. Experiments were conducted at a laboratory of Toyo University.

\subsection{Comparison between conventional and proposed method}

Fig. 3 shows the result of the conventional and proposed method in the individual TCP transmission, and the characteristic part is extracted from the data of 180 seconds. From the top, the results of FD throughput with unit time $1 \mathrm{msec}$, FN throughput when $K=1$ and OD throughput. The horizontal axis is time and the left vertical axes are throughputs of TCP Data and Signal, and the right them are those of TCP ACK. Black line is the throughputs of TCP Data, Red is those of TCP ACK, and Green filling for clarity is that of Signal. First, the evaluation of FD throughput in Fig. 3(a) is rough, and the detailed communication situation cannot be confirmed. As shown in previous study [1], it is evaluated only by how many packets are within the unit time. Fig. 3(b) shows the evaluation of FN throughput. Wireshark put a timestamp at the end of the packet, so the preamble start time was calculated backwards from there to be the start time. It is similar to the proposed method in terms of throughput evaluation on a per-packet basis. Since the width of the rectangle is the transmission time of one packet, the area represents the communication traffic volume. Unlike in FD throughput, the order relationship between TCP data and ACK can be visualized. Furthermore, the difference in FN throughput indicates the difference in the radio transmission speed. In the proposed method, Fig. 3(c), the width of the rectangle is Occupied Duration and its area is the communication traffic volume. However, there is no gap between the rectangles and if there was a gap, it is Idle Time as defined in Sect. 2.3. Since Occupied Duration also includes a variable Back-off Time, as shown in dotted line A, the difference in values can be expressed as a difference in OD throughput. The proposed method defines the evaluation method of Signals in detail. Therefore, it is able to evaluate Signals at the time of the blank in FN throughput, while the previous study has not been able to identify the reason of the failure of packet transmission. Furthermore, the same throughput at lower values appears in B and C. This is a situation where TCP DATA and ACK are interrupted when retransmissions occur, as defined by Sect. 2.4. The proposed method evaluates the throughput by the time when a packet communication is successfully completed, that is, Occupied Duration. From the above comparison, the proposed method not only evaluates the throughput, but also can evaluate in 

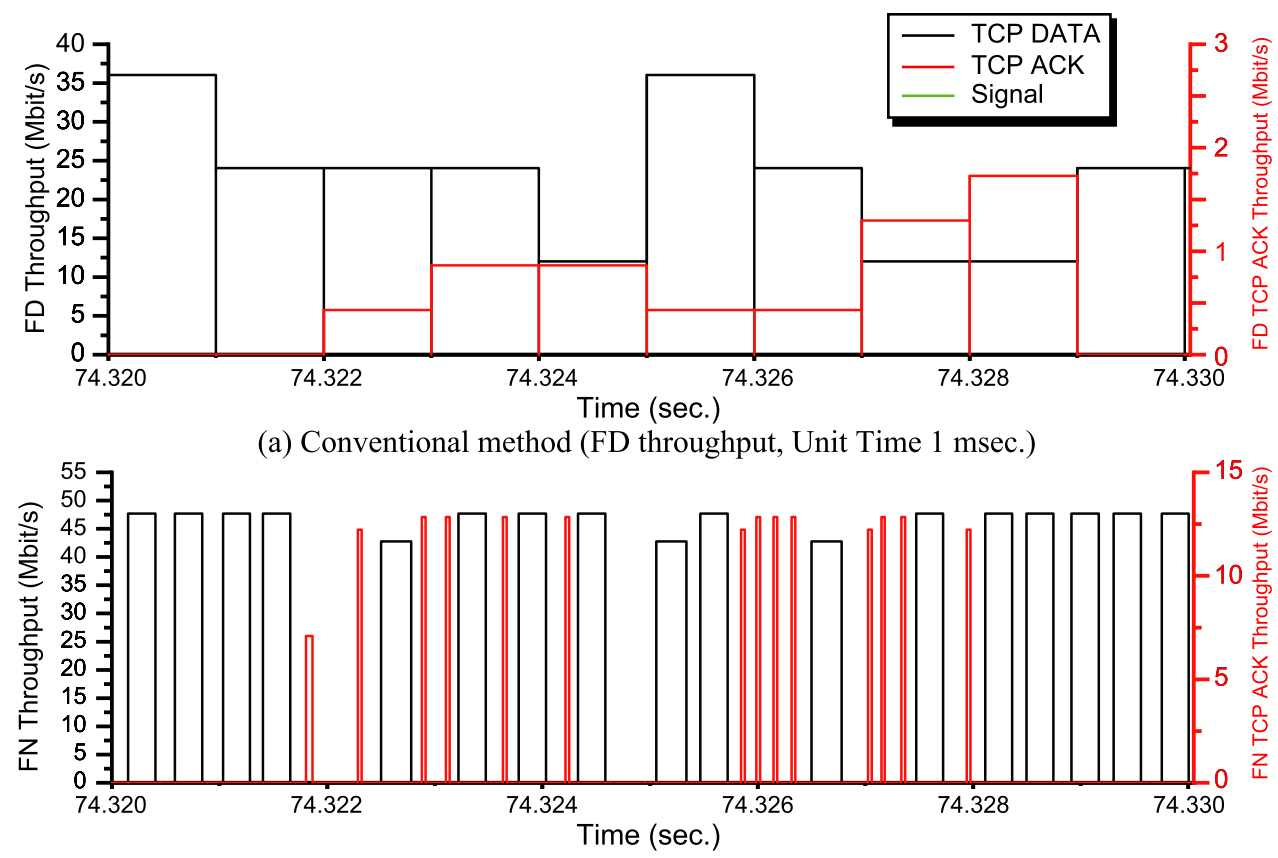

(b) Conventional method (FN throughput, when $K=1$ )

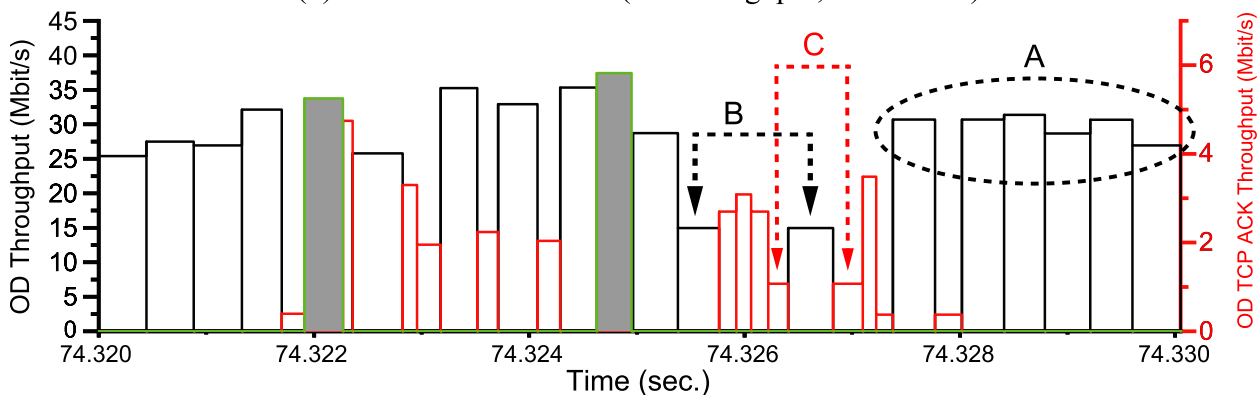

(c) Proposed method

Fig. 3. Application and analysis example.

more detail whether the decrease is due to the condition of the communications path or to competing packets.

\section{Conclusion}

We proposed OD throughput based on Occupied Duration in individual TCP transmission. From the comparison with the conventional methods, the effectiveness was confirmed. As further study, we will extent to $802.11 \mathrm{n}$ or ac mode that needs to consider frame aggregation etc., UDP and TCP competitive transmissions. 